\title{
Estudo da relação entre a geração de resíduos sólidos urbanos e o Produto Interno Bruto (PIB) per-capito no Brasil
}

\author{
Study of the relationship between the generation of solid urban waste and the Gross Domestic \\ Product (GDP) per capita in Brazil
}

Estudio de la relación entre la generación de residuos sólidos urbanos y el Producto Interno Bruto (PIB) per cápita en Brasil

\author{
Alison de Souza Norberto \\ ORCID: https://orcid.org/0000-0002-4303-4847 \\ Universidade Federal de Pernambuco, Brasil \\ E-mail: alison_norberto@hotmail.com \\ Silas Almeida Lira \\ ORCID: https://orcid.org/0000-0001-6428-7029 \\ Autarquia Educacional do Belo Jardim, Brasil \\ E-mail: silas_al@hotmail.com \\ Anderson Viana do Nascimento \\ ORCID: https://orcid.org/0000-0001-7117-3054 \\ Universidade Federal de Pernambuco, Brasil \\ E-mail: andersonvnascimento@ outlook.com \\ Armando Dias Duarte \\ ORCID: http://orcid.org/0000-0003-2571-7705 \\ Universidade Federal de Pernambuco, Brasil \\ E-mail: armando01.dias@gmail.com \\ Jailma Gabrieli Cavalcante da Silva \\ ORCID: https://orcid.org/0000-0002-0326-4314 \\ Autarquia Educacional do Belo Jardim, Brasil \\ E-mail: jailmagabrieli4@gmail.com \\ João Victor Cavalcanti Alves \\ ORCID: https://orcid.org/0000-0002-7766-2980 \\ Autarquia Educacional do Belo Jardim, Brasil \\ E-mail: jcavalvanti900@ gmail.com \\ Thayse Diniz Pedrosa \\ ORCID: https://orcid.org/0000-0002-5769-7668 \\ Universidade Federal de Pernambuco, Brasil \\ E-mail: thaysediniz1@hotmail.com \\ José Francisco de Oliveira Neto \\ ORCID: https://orcid.org/0000-0002-4986-2643 \\ Universidade Federal de Pernambuco, Brasil \\ E-mail: neto_fbo@hotmail.com
}

\begin{abstract}
Resumo
A redução da geração de resíduos sólidos no Brasil vem sendo abordada ao longo de décadas, no ano de 2010 com o lançamento da Política Nacional de Resíduos Sólidos (PNRS) viu-se então diretrizes mais sólidas para promover essa redução. No que concerne ao processo de geração de resíduos sólidos urbanos (RSU), diversos aspectos estão relacionados, dentre os quais, os aspectos econômicos. Diante deste cenário, esta pesquisa buscou avaliar a relação da geração de RSU per capita do Brasil com o seu Produto Interno Bruto (PIB) per capito ao longo das duas primeiras décadas do século XXI. Nas análises, foram utilizados os dados dos relatórios da Associação Brasileira de Empresas de Limpeza Pública e Resíduos Especiais (ABRELPE) e dados do PIB divulgados pelo governo federal. Ao fim, identificou-se que os indicadores não estão correlacionais quando do ponto de vista geral do país, mas que trazem um comportamento diferente em algumas de suas regiões, que sofreram a influência de fatores econômicos resultando em um comportamento diferente do resultado nacional.
\end{abstract}

Palavras-chave: Geração de resíduos; Aspectos econômicos; Comportamento; Correlacionais.

\begin{abstract}
The reduction of solid waste generation in Brazil has been addressed for decades, in 2010 with the launch of the National Solid Waste Policy (PSWP), we saw more solid guidelines to promote this reduction. Regarding the process of generating solid urban waste (MSW), several aspects are related, among which, the economic aspects. Given this
\end{abstract}


scenario, this research sought to assess the relationship between the generation of MSW per capita in Brazil and its Gross Domestic Product (GDP) per capita over the first two decades of the 21 st century. The data from the reports of the Brazilian Association of Public Cleaning Companies and Special Waste (ABRELPE) and GDP data released by the federal government were used in the analyzes. At the end, it was identified that the indicators are not correlational when from the general point of view of the country, but that it brings a different behavior in some of its regions, which were influenced by economic factors resulting in a different behavior from the national result.

Keywords: Generation of waste; Economic aspects; Behavior; Correlational.

\section{Resumen}

La reducción de la generación de residuos sólidos en Brasil se ha abordado durante décadas, en 2010 con el lanzamiento de la Política Nacional de Residuos Sólidos (PNRS), vimos lineamientos más sólidos para promover esta reducción. En cuanto al proceso de generación de residuos sólidos urbanos (RSU), se relacionan varios aspectos, entre los cuales, los económicos. Ante este escenario, esta investigación buscó evaluar la relación entre la generación de RSU per cápita en Brasil y su Producto Interno Bruto (PIB) per cápita durante las dos primeras décadas del siglo XXI. En los análisis se utilizaron los datos de los informes de la Asociación Brasileña de Empresas Públicas de Limpieza y Residuos Especiales (ABRELPE) y los datos del PIB publicados por el gobierno federal. Al final, se identificó que los indicadores no son correlacionales cuando desde el punto de vista general del país, sino que trae un comportamiento diferente en algunas de sus regiones, las cuales fueron influenciadas por factores económicos resultando en un comportamiento diferente al resultado nacional.

Palabras clave: Generación de residuos; Aspectos económicos; Comportamiento; Correlacional.

\section{Introdução}

Um problema enfrentado por países em desenvolvimento, como o Brasil, é a imperícia dos serviços de limpeza urbana em coletar e destinar de forma adequada, do ponto de vista ambiental, aos resíduos sólidos produzidos nos diversos setores da sociedade. Em consequência, grande parte dos resíduos gerados não são coletados regularmente, permanecendo junto às habitações, em especial nas áreas de baixa renda, ou acabam sendo dispostos em áreas inadequadas, como logradouros públicos, terrenos baldios, encostas e até cursos d'água. Os lixões são a forma mais inadequada de acomodação dos resíduos, um lugar a céu aberto trazendo risco ao sistema de saúde brasileiro, de acordo com a Associação Brasileira de Empresas de Limpeza Pública e Resíduos Especiais (Abrelpe, 2019).

A Associação Brasileira de Normas Técnicas (ABNT, 2004) define os resíduos sólidos como:

Resíduos nos estados sólido e semissólido, que resultam de atividades de origem industrial, doméstica, hospitalar, comercial, agrícola, de serviços e de varrição. Ficam incluídos nesta definição os lodos provenientes de sistemas de tratamento de água, aqueles gerados em equipamentos e instalações de controle de poluição, bem como determinados líquidos cujas particularidades tornem inviável o seu lançamento na rede pública de esgotos ou corpos de água, ou exijam para isso soluções técnica e economicamente inviáveis em face à melhor tecnologia disponível (ABNT, 2004, p. 1).

Quanto a sua origem, os resíduos sólidos podem ser classificados em Resíduos Sólidos Urbanos (RSU), Resíduos Industriais, Resíduos de Serviços de Saúde (RSS), resíduos de portos, aeroportos, terminais rodoviários e ferroviários (compreendem os resíduos gerados tanto nos terminais como dentro dos navios, aviões e veículos de transporte), resíduos agrícolas, Resíduos de Construção e Demolição (RCD) e resíduos radioativos. Sousa et al. (2016) apontam que essa classificação facilita o gerenciamento mais eficaz dos resíduos, pois facilita o monitoramento e a sinalização particular de um planejamento bem orientado.

Nas décadas de 80 e 90, grande parte dos municípios brasileiros apresentavam características muito semelhantes, da geração à disposição final, quanto ao manejo dos resíduos sólidos, abrangendo apenas as atividades de coleta regular, transporte e descarga final. Com a implementação da Lei No 12.305/10, que institui a Política Nacional de Resíduos Sólidos (PNRS), alguns municípios passaram a incluir outros procedimentos na sua rota de manejo dos resíduos sólidos, tais como coleta seletiva, reciclagem, compostagem e incineração. 
A PNRS foi o marco importante na gestão e gerenciamentos dos resíduos sólidos no país, estabelecendo a seguinte ordem de prioridade: não geração, redução, reutilização, reciclagem, tratamento de resíduos sólidos e disposição ambientalmente adequada dos rejeitos (Brasil, 2010). A legislação trouxe uma abordagem sobre novos conceitos, como acordo setorial, sustentabilidade operacional e financeira, integração dos catadores de recicláveis, padrões sustentáveis de produção e consumo, logística reversa, entre outros (Jucá et al., 2014).

No decorrer dos anos, os municípios brasileiros estiveram em crescente urbanização e industrialização, estabelecendo um grande fluxo de pessoas e aumentando instantaneamente o consumo. Isso influenciou diretamente o aumento de resíduos sólidos principalmente em grandes centros urbanos, trazendo risco à população local, pois diariamente são coletadas milhares de toneladas, havendo em sua composição elementos perigosos ao ecossistema e à saúde humana.

Em 2018, no Brasil, foram geradas 79 milhões de toneladas de resíduos. Um aumento de pouco menos de $1 \%$ em relação ao ano anterior. Desse montante, 92\% (72,7 milhões) foi coletado. De acordo com a ABRELPE (2019), isso significa uma alta de 1,66\% em comparação a 2017. Ou seja, a coleta aumentou num ritmo um pouco maior que a geração. Por outro, evidencia que 6,3 milhões de toneladas de resíduos não foram recolhidas junto aos locais de geração. A destinação adequada em aterros sanitários recebeu 59,5\% dos resíduos sólidos urbanos coletados, o que equivale a 43,3 milhões de toneladas, um pequeno avanço em relação ao cenário do ano anterior. Os 40,5\% restantes foram despejados em locais inadequados por 3.001 municípios (Abrelpe, 2019).

As quantidades de resíduos sólidos têm crescido constantemente junto com o Produto Interno Bruto (PIB) nas últimas décadas. Por exemplo, a quantidade total de resíduo municipal per capita aumentou 29\% no Norte América, 35\% na Organização para a Cooperação e Desenvolvimento Econômico (OCDE) e 54\% na União Europeia (UE) de 1980 a 2005 (Sjöström \& Östblom, 2010).

Segundo Martins \& Stein (2014), o aumento da geração de resíduos sólidos tem como principais causas a variação no tamanho da população e o aumento do produto interno bruto. No Brasil, entre os anos de 2001 a 2010, a taxa média de crescimento populacional foi de $1,17 \%$ ao ano, correspondendo $12,3 \%$ na última década. O PIB teve um aumento de 3,6\% ao ano e o PIB per capita médio foi de 2,4\%, mostrando assim que o Brasil esteve em um progressivo crescimento na década passada (IBGE, 2010).

Neste contexto, o presente estudo buscou analisar a relação dos aspectos econômicos com a geração de resíduos sólidos no país, por meio de pesquisas bibliográficas e documentais, para assim contribuir nas análises e perspectivas que projetam a redução da geração de resíduos sólidos e os impactos econômicos que isto pode promover.

\section{Metodologia}

A pesquisa foi categorizada como quantitativa pois, de acordo com Pereira et al. (2018), os métodos quantitativos, após a coleta de dados, podem ser analisados por técnicas estatísticas como por exemplo: estatísticas, porcentagens, métodos numéricos, métodos analíticos etc. O enfoque adotado foi o estatístico, que, de acordo com Lakatos \& Marconi (2003), fornece uma descrição quantitativa da sociedade e que posteriormente é possível medir a importância, a variação ou qualquer outro atributo que possa ser quantificado que contribua para o entendimento da pesquisa.

A pesquisa consistiu em três etapas, a primeira delas, o levantamento dos dados de geração e análise de correlações estatísticas, onde foram definidos as variáveis e o intervalo de tempo dos dados a serem realizadas, decidindo-se adotar o intervalo de tempo entre 2007-2018 (11 anos). Após a definição do intervalo de tempo de análise, a segunda etapa consistiu em realizar a coleta dos dados da geração per capita dos resíduos sólidos no Brasil, e do PIB per capita nesse intervalo de tempo de 11 anos. 
Os dados da geração per capita de resíduos foram obtidos por meio dos relatórios anuais da ABRELPE (2019), que divulga anualmente desde o ano de 2003 um Panorama dos Resíduos Sólidos no Brasil. Esse Panorama tem o objetivo de facilitar o acesso dos órgãos governamentais, das empresas públicas e privadas, das organizações não-governamentais, entidades educativas, da imprensa e da sociedade em geral, às informações sobre os resíduos sólidos em seus diversos segmentos, que em muitos casos estão fracionadas e/ou desatualizadas.

Os dados do PIB foram coletados de três fontes: Deepask (2015), Instituto Brasileiro de Geografia e Estatística IBGE (2020) e Valor Econômico (2018).

Após a coleta dos dados, a terceira etapa do trabalho foi efetuar análises de correlações estatísticas dos PIB e da geração per capita no intervalo de tempo estabelecido pelo estudo. Para tal, foi utilizado o software Minitab® (Coutinho, 2017), que foi criado com o objetivo de ser um facilitador do processo de tomada de decisões pautadas em análises e estudos estatísticos.

Também foram verificados os graus de correlação do PIB per capito nacional e das cinco grandes regiões do país: Norte, Nordeste, Centro-Oeste, Sul e Sudeste (Figura 1), com a geração de resíduos sólidos per capita da respectiva região.

Figura 1 - Representação gráfica das cinco regiões do Brasil.

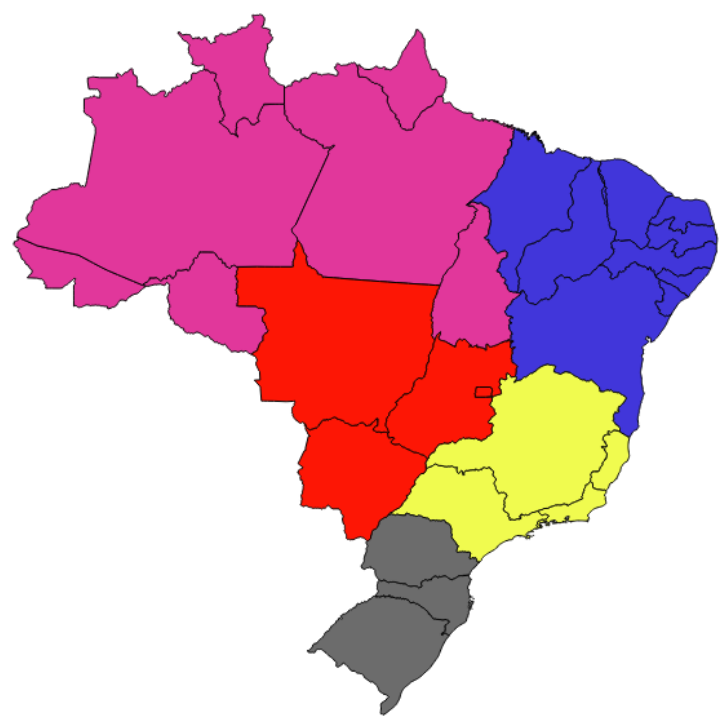

\begin{tabular}{|l|l|}
\hline & Norte \\
\hline & Nordeste \\
\hline & Centro-Oeste \\
\hline & Sudeste \\
\hline & Sul \\
\hline
\end{tabular}

Fonte: Autores (2020).

A formulação para o cálculo da correlação entre as duas variáveis arbitrárias expressas pela Equação 1.

$$
\rho(\mathrm{x}, \mathrm{y})=\left[\sum_{i=1}^{n}(\mathrm{xi} * \mathrm{yi})-n *(\bar{x}-\bar{y})\right] /\left[\left(\sqrt{ } \sum_{i=1}^{n}(\mathrm{xi}-\bar{x})^{2}\right) *\left(\sqrt{ } \sum_{i=1}^{n}(\mathrm{yi}-\bar{x})^{2}\right] \quad \text { (Eq. } 1\right)
$$

Onde:

$\rho(x, y)=$ correlação entre variáveis;

$\bar{x}=$ média aritmética da variável $\mathrm{x}$;

$\mathrm{x}_{\mathrm{i}}=$ valor da variável na observação $i$;

$\bar{y}=$ média aritmética da variável y;

$\mathrm{y}_{\mathrm{i}}=$ valor da variável na observação $\mathrm{i}$;

$\mathrm{n}=$ número de observações.

A variável de correlação, $\rho(x, y)$, possui variação entre -1 e 1 , quanto mais próximo de 1 o resultado indicará uma correlação forte e positiva, evidenciando assim a existência de uma correlação entre as variáveis, isso significa dizer que 
quando uma variável cresce a outra também cresce, da mesma forma para o decréscimo destas variáveis. Já para os valores de correlação próximos de -1 , o resultado indica a existência de uma correlação forte e negativa, evidenciando que quando uma variável cresce a outra decresce e vice-versa. Para valores de correlação próximos de 0 , a indicação é de provável inexistência de correlação entre as variáveis analisadas.

Na Figura 2 são apresentadas as formas mais convencionais de comportamento de correlações gráficas.

Figura 2 - Exemplo da dispersão gráfica para comportamentos da correlação $\rho(x, y)$.
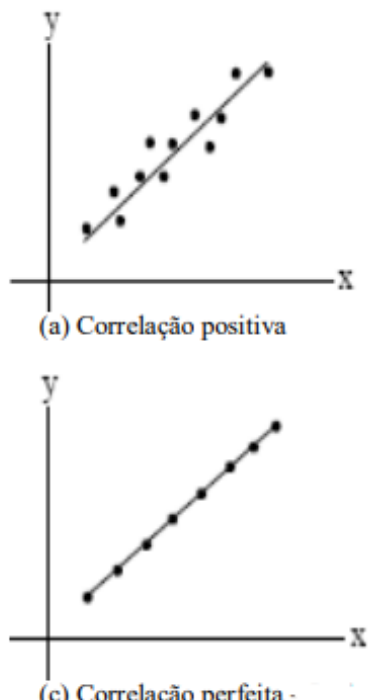

Fonte: Spiegel et al. (2016).
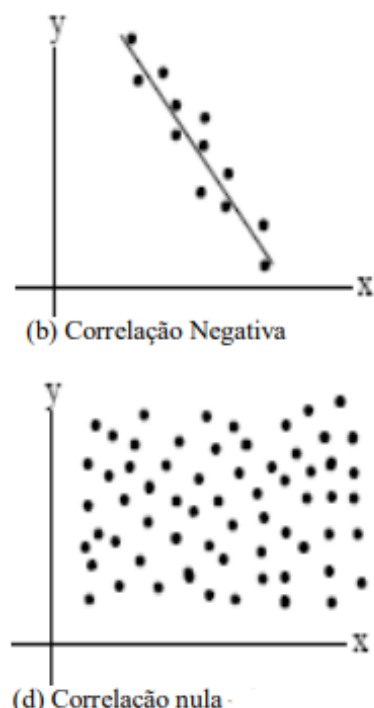

(d) Correlação nula

\section{Resultados e Discussões}

Após coleta de dados e a inserção no software, foi realizada a análise em duas esferas, uma primeira no âmbito nacional e outra no âmbito regional do país (Norte, Nordeste, Centro-Oeste, Sudeste e Sul).

\section{Nacional:}

Na Figura 3 são apresentados os dados coletados na literatura referentes a geração per capita de RSU e do PIB no Brasil entre os anos 2007 e 2018. Foi realizada a análise de regressão no software Minitab®, para verificar a correlação entre os indicadores quanto aos dados no Brasil (Figura 4). 
Figura 3 - Geração de resíduos per capita x PIB per capita no Brasil (2007 a 2018).

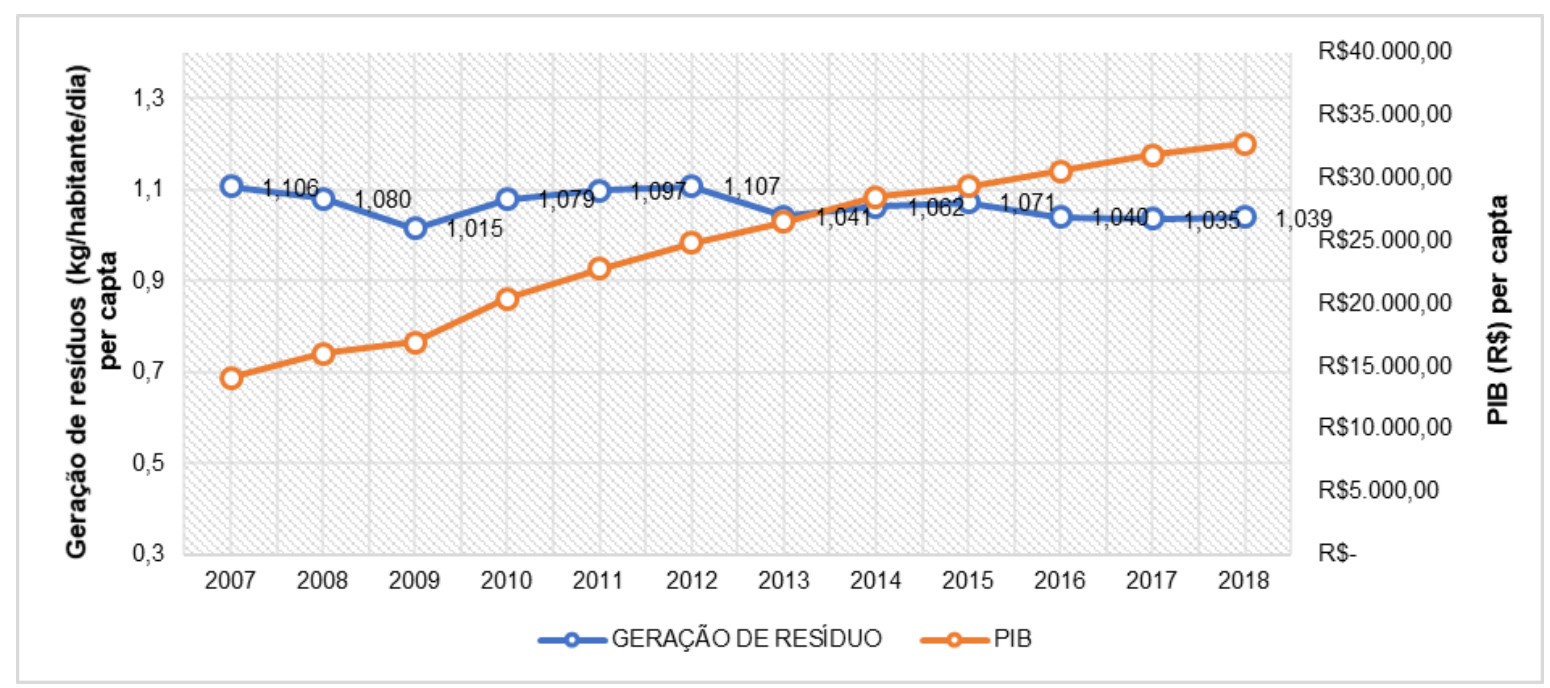

Fonte: Autores (2020).

Figura 4 - Análise de correlação entre a geração de resíduo x PIB entre os anos 2007 de 2018 no Brasil: (a) Função de correlação entre as variáveis; (b) Grau de correlação.

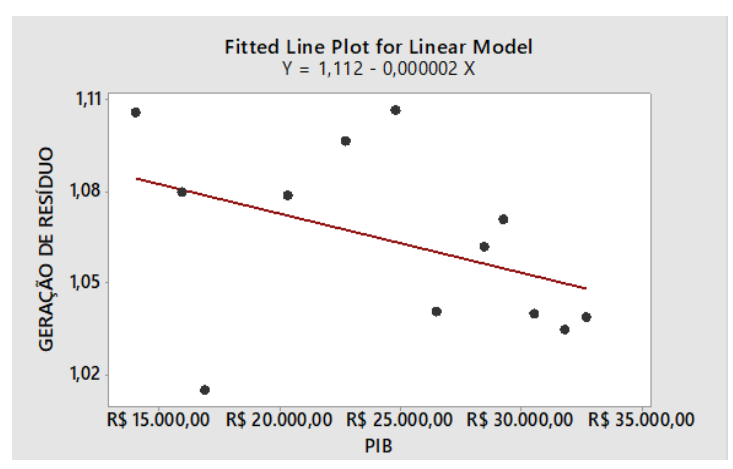

(a)

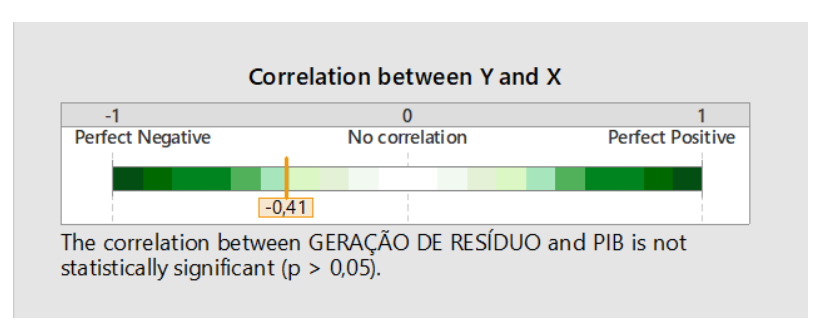

(b)

Fonte: Autores (2020).

Quanto à evolução histórica do PIB per capito no Brasil, é possível notar um crescimento linear ao longo do período avaliado. Quanto à geração per capita de resíduos, seu comportamento foi praticamente constante na análise temporal.

$\mathrm{Na}$ análise a nível nacional os dados mostram que estatisticamente não existe correlação entre os indicadores, dado o valor da correlação, -0,41 (Figura 4, item b), sendo que valores de correlação considerados altos quando próximos 1 ou -1.

Segundo Sjöström \& Östblom (2010) as tecnologias e o comportamentos das famílias diferem, no que diz respeito às intensidades de geração de resíduos e de suas atividades econômicas. Esse aspecto pode ser notado analisando hábitos e atividades separadamente as cinco grandes regiões do Brasil, por exemplo, comparando as principais atividades econômicas das regiões sudeste e do centro-oeste do Brasil, no sudeste do país há a maior concentração industrial, já na região centro-oeste as principais atividades econômicas são derivadas do setor do agronegócio. Esse aspecto incide diretamente no tipo e na quantidade de resíduos gerado em cada região.

Além disso, Resende \& Magalhães (2013) discutem que a heterogeneidade regional brasileira é marcante e persistente ao longo das décadas, caracterizada pela própria extensão territorial. Em países com dimensões continentais como Brasil, com população de 211.755 .692 habitantes e área de $8.516 .000 \mathrm{~km}^{2}$, as análises correlacionais devem ser trabalhadas incorporando o 
efeito regional de seus fatores (IBGE, 2020). A fim disso, nos itens seguintes são apresentadas as análises correlacionais entre geração de resíduos e PIB para as cinco grandes regiões do Brasil.

\section{Região Norte}

De acordo com o IBGE (2020) a região Norte do Brasil possui população de 18.672.591 habitantes e área de $3.870 .000 \mathrm{~km}^{2}$. Segundo Oliveira et al. (2019) a situação da destinação de resíduos sólidos na região norte é preocupante por ser um ambiente amazônico, com altas taxas de pluviométricas, altas temperaturas, predominância de logística via hidrovia e outros fatores.

Somado aos aspectos climáticos e territoriais, os dados da Programa das Nações Unidas para o Desenvolvimento PNUD (2018), apontam que a região Norte possuía um IDH de 0,667, em um intervalo de 0 a 1 , este parâmetro está relacionado as condições econômicas, educacionais, de saúde e dos serviços de saneamento, que neste contexto está diretamente vinculada as condições de como a região trata, coleta, destina seus resíduos e das políticas desenvolvidas no tratamento.

Ciente do contexto do cenário apresentado para a região Norte na Figura 5 são apresentados os dados coletados na literatura técnica referentes a geração per capita de RSU e do PIB per capito na região Norte do Brasil entre os anos 2007 a 2015, entre os anos 2016-2018 não foram encontrados os dados do PIB por região. Dos dados da Figura 5 foi realizada uma análise de regressão linear para verificar o grau de correlação das variáveis, demonstrada na Figura 6.

Figura 5 - Geração de resíduos per capita x PIB per capita na região Norte (2007 a 2015).

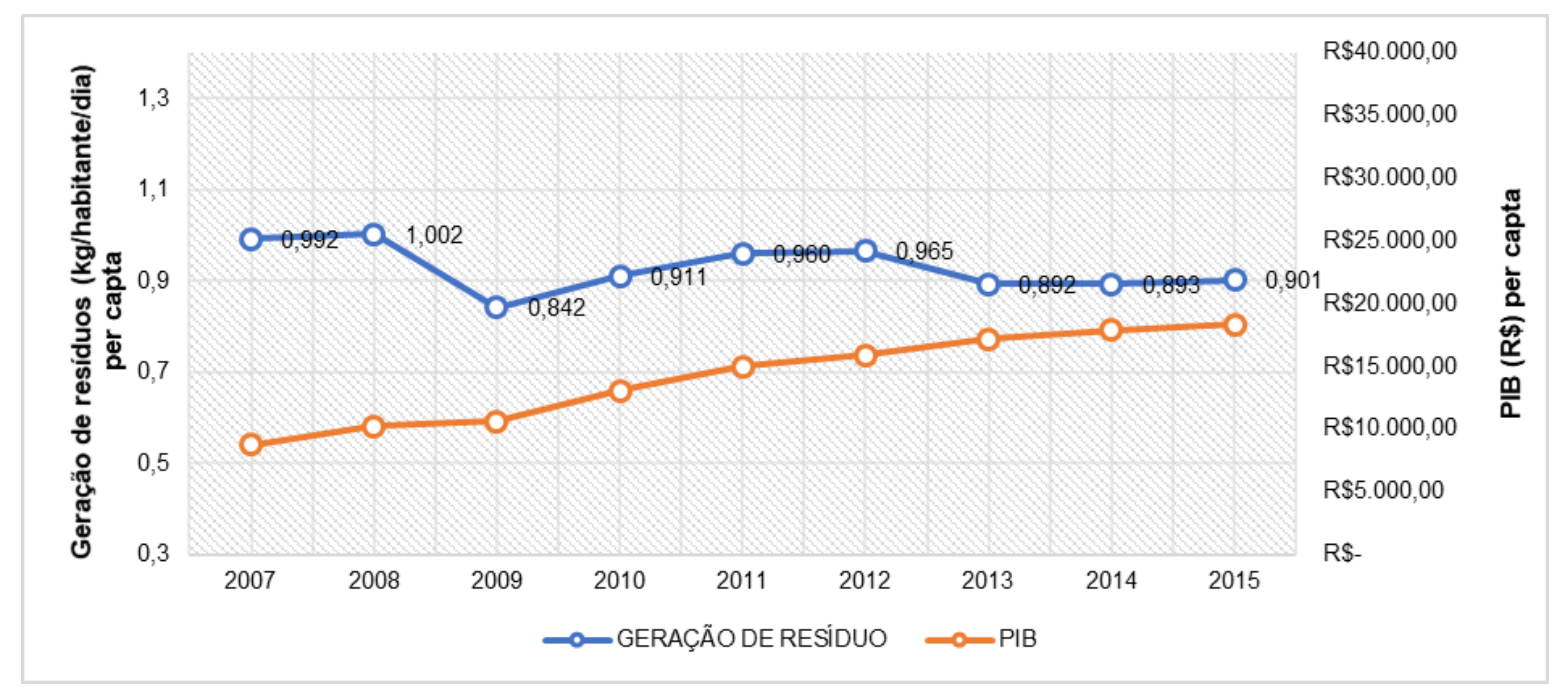

Fonte: Autores (2020). 
Figura 6 - Análise de correlação entre a geração de resíduo x PIB entre os anos 2007 e 2015 na região Norte: (a) Função de correlação entre as variáveis; (b) Grau de correlação.

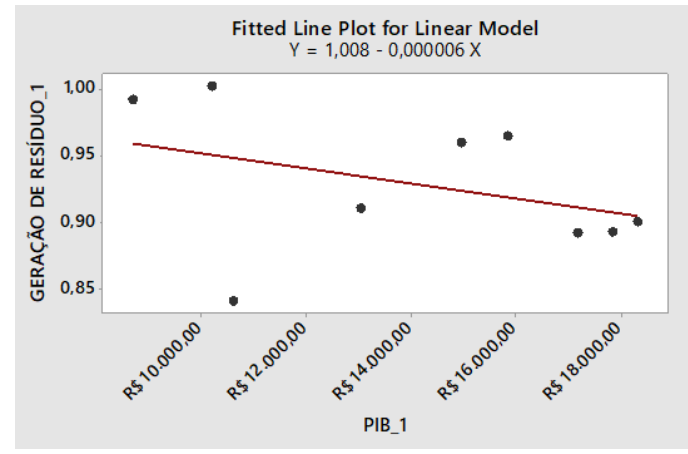

(a)

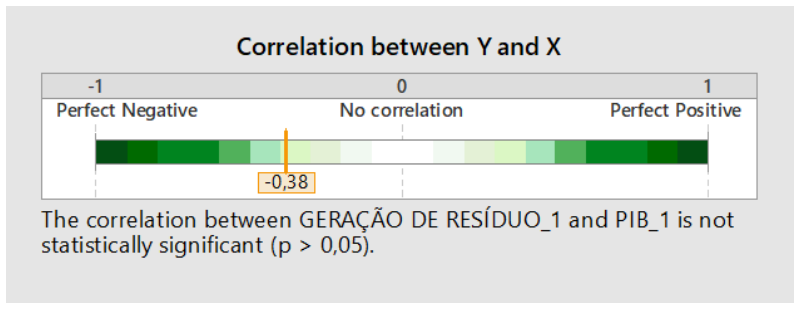

(b)

Fonte: Autores (2020).

Quanto à análise da geração per capita de resíduos na região Norte, foi verificada uma baixa oscilação de valores no período analisado, faixa que variou entre 0,842 e 1,002. Já o PIB per capito apresentou-se de forma crescente ao longo do tempo. Da análise de correlação, constatou-se que para a região Norte não existe correlação estatística entre as duas variáveis analisadas, tendo em vista o baixo valor da correlação, de -0,38 (Figura 6, item b).

\section{Região Nordeste}

O Nordeste do Brasil apresenta-se como uma vasta região em termos geográficos, econômicos e demográficos, superpovoada, apesar da imagem que se faz nos centros mais desenvolvidos do país, possui grande importância econômica e social (Sousa et al., 2017). De acordo com o IBGE (2020), o Nordeste possui 57.374.243 habitantes e área de 1.554 .000 km². Esses números fazem com que o Nordeste seja a segunda região mais populosa do país, superada somente pela região Sudeste.

Segundo dados da PNUD (2018), a região Nordeste possuía um IDH de 0,663, menor valor entre as cinco grandes regiões do Brasil. Segundo Sousa et al. (2017), as secas na região prejudicam, sobretudo, as camadas sociais mais pobres, que não dispõe de recursos suficientes para sobreviverem aos períodos de longa estiagem. Consequentemente, essas camadas sociais perdem tudo que possuem e são forçadas a migrar para o centro-sul, o que fornece momentos oportunos para os grandes proprietários adquirirem as terras e o gado dos agricultores que não têm condições de alimentar seus animais e de permanecer em suas terras. Essa é a chamada indústria da seca, que favorece os grupos econômicos e políticos que a controlam, inviabilizando as políticas criadas para amenizar os efeitos das estiagens.

Diante dos aspectos da região Nordeste apresentados, na Figura 7, são apresentados os dados coletados na literatura técnica, referentes à geração per capita de RSU e do PIB na região entre os anos 2007 a 2015. E a partir dos dados do PIB e geração per capita de resíduos foi realizada uma análise de regressão linear para verificar o grau de correlação das variáveis (Figura 8). 
Figura 7 - Geração de resíduos per capita x PIB per capita na região Nordeste (2007 a 2015).

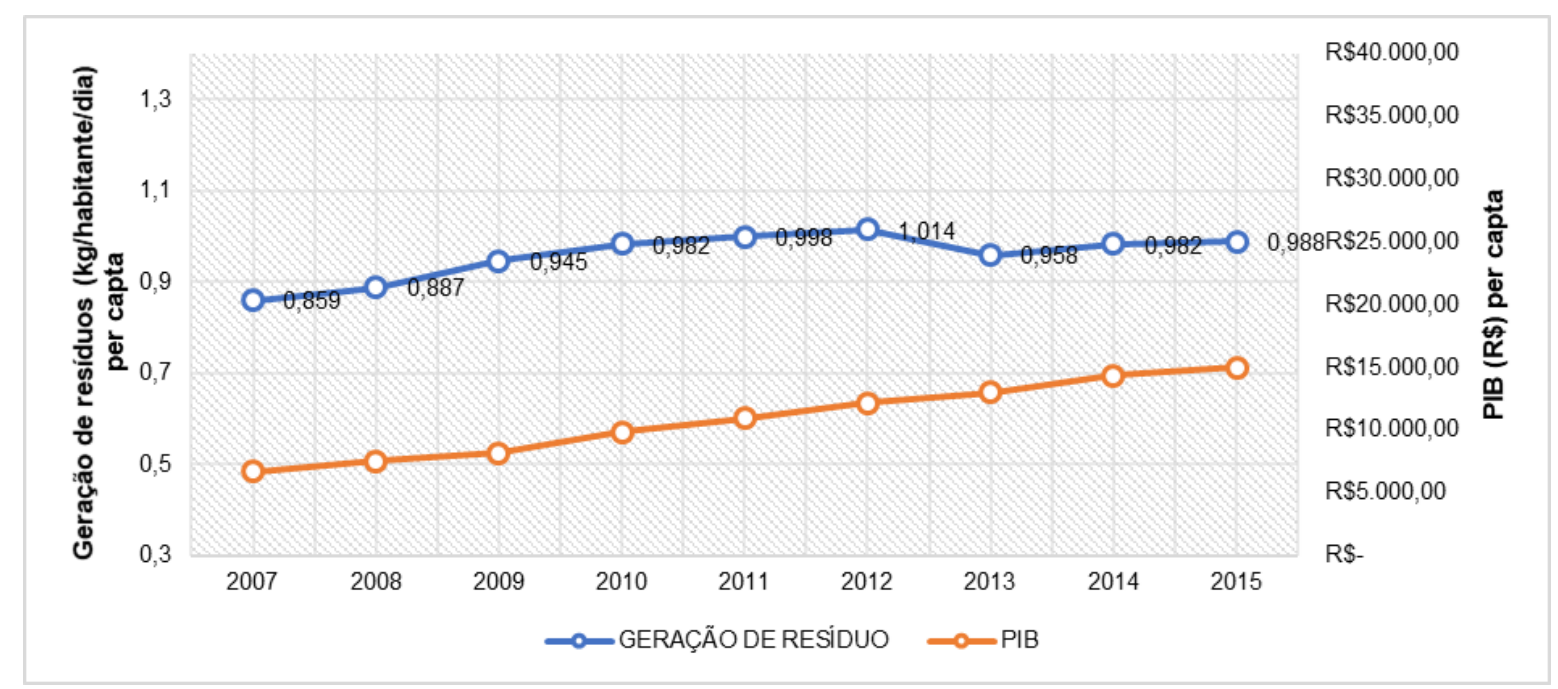

Fonte: Autores (2020).

Figura 8 - Análise de correlação entre a geração de resíduo x PIB entre os anos de 2007 e 2015 na região Nordeste: (a) Função de correlação entre as variáveis; (b) Grau de correlação.

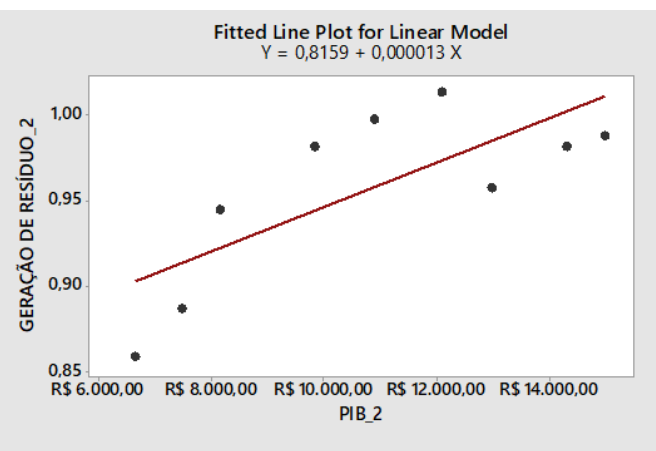

(a)

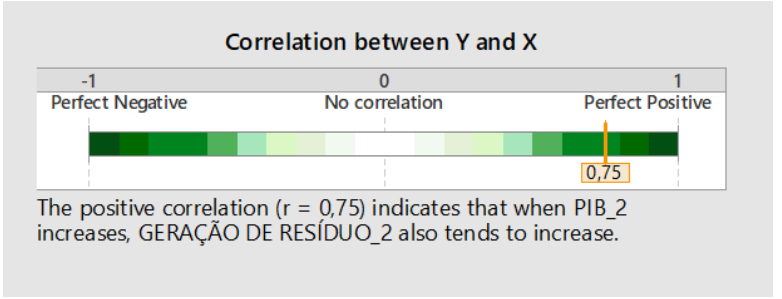

(b)

Fonte: Autores (2020).

Os dados do período de 2007 a 2015 mostram que ambos os parâmetros apresentaram crescimento no intervalo de tempo (Figura 8, item a). Esse crescimento teve efeito no cálculo do grau de correlação, o qual apresentou um valor de 0,75 (Figura 8 , item b), que indica a existência de correlação positiva das variáveis PIB e geração de resíduos per capito na região Nordeste.

\section{Região Centro-Oeste}

De acordo com IBGE (2020), a região Centro-Oeste do Brasil possui população de 16.504.303 habitantes e área de 1.612.000 km². Segundo dados da PNUD (2018), a região possuía um IDH de 0,757, segundo maior índice das regiões do Brasil ficando atrás apenas da região Sudeste.

O Centro-Oeste tem sido, nas últimas quatro décadas, a região com melhor desempenho econômico entre as macrorregiões brasileiras. Sua participação no PIB nacional, que em 1970 era de 3,8\%, passou para os cerca de $10 \%$ atuais, o equivalente a $\mathrm{R} \$ 480$ bilhões. A região obteve um excepcional avanço da atividade agropecuária nas últimas décadas, mas permanece com um baixíssimo grau de industrialização, respondendo por cerca de $4 \%$ da produção industrial nacional, percentual muito inferior à participação da região no PIB nacional, de cerca de 10\% (MIRAGAYA, 2014).

Ciente do panorama da região centro-oeste, na Figura 9, são apresentados os dados da região Centro-Oeste do Brasil 
entre os anos 2007 a 2015. Os dados estatísticos são demonstrados na Figura 10.

Figura 9 - Geração de resíduos per capita x PIB per capita na região Centro-Oeste (2007 a 2015).

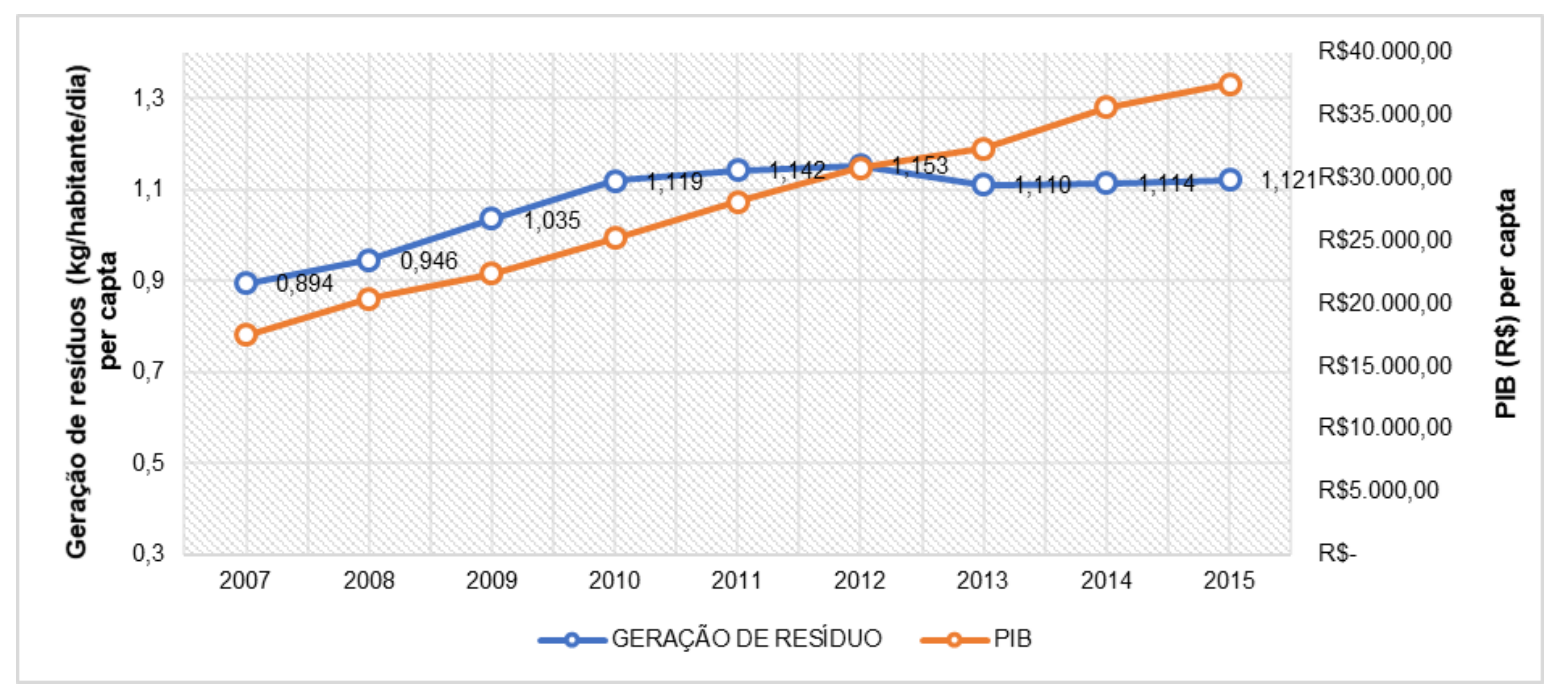

Fonte: Autores (2020).

Figura 10 - Análise de correlação entre a geração de resíduo x PIB entre os anos de 2007 e 2015 na região Centro-Oeste: (a) Função de correlação entre as variáveis; (b) Grau de correlação.

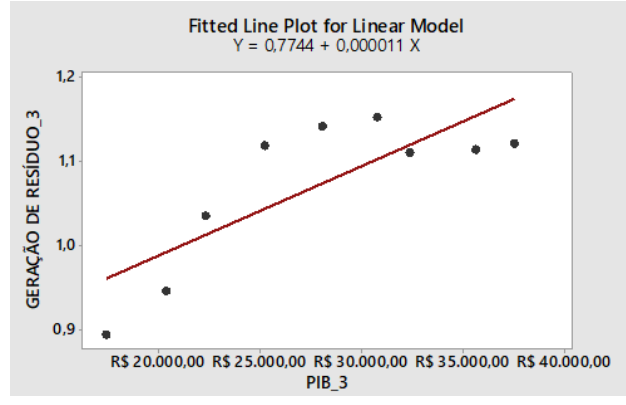

(a)

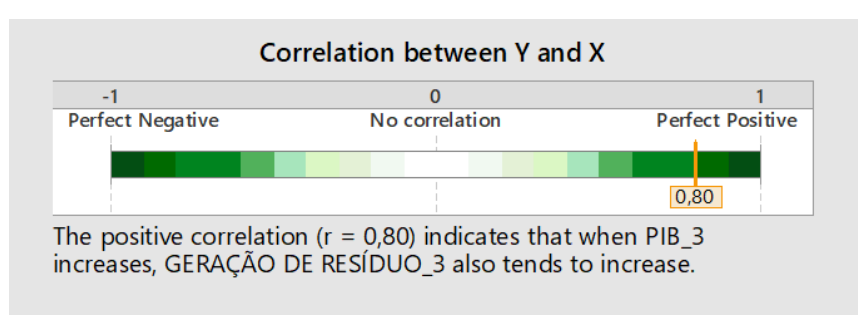

(b)

Fonte: Autores (2020).

Quanto à geração per capita de resíduos, entre os anos de 2007 e 2010, podê-se perceber um acentuado crescimento, se mantendo praticamente constante nos anos seguintes. Dos dados do PIB per capito foi verificado um acentuado crescimento ao longo de todo período de análise, sendo o maior crescimento entre as regiões já apresentadas (Figura 10, item a). Quanto à correlação estatística, os dados mostram que estatisticamente existe correlação entre os indicadores, tendo em vista o valor de correlação linear de 0,80 (Figura 10, item b), que indica que as variáveis se correlacionam de forma positiva, o que significa dizer que à medida que uma cresce a outra também cresce.

\section{Região Sudeste}

Segundo dados IBGE (2020), a região Sudeste do Brasil possui população de 89.012.240 habitantes, região com maior densidade populacional do país, e área de $924.511 \mathrm{~km}^{2}$. Segundo dados da PNUD (2018), a região possuía um IDH de 0,766, maior índice entre as cinco regiões do país, indicativo de que a região é a que possui os melhores índices de qualidade de vida do Brasil.

A região Sudeste do Brasil, formada pelos estados de São Paulo, Rio de Janeiro, Minas Gerais e Espírito Santo, constitui-se como a região mais importante do país no aspecto econômico. Essa importância reside no fato de que nesta região 
estão localizados os maiores centros urbanos do país, o maior volume de riqueza produzido e os maiores mercados consumidores (SILVA \& CAIRES, 2019).

Na Figura 11 são apresentados os dados coletados na literatura técnica referentes a geração per capita de RSU e do PIB na região Sudeste do Brasil entre os anos 2007 a 2015. A Figura 12 indica os resultados estatísticos.

Figura 11 - Geração de resíduos per capita x PIB per capita na região Sudeste (2007 a 2015).

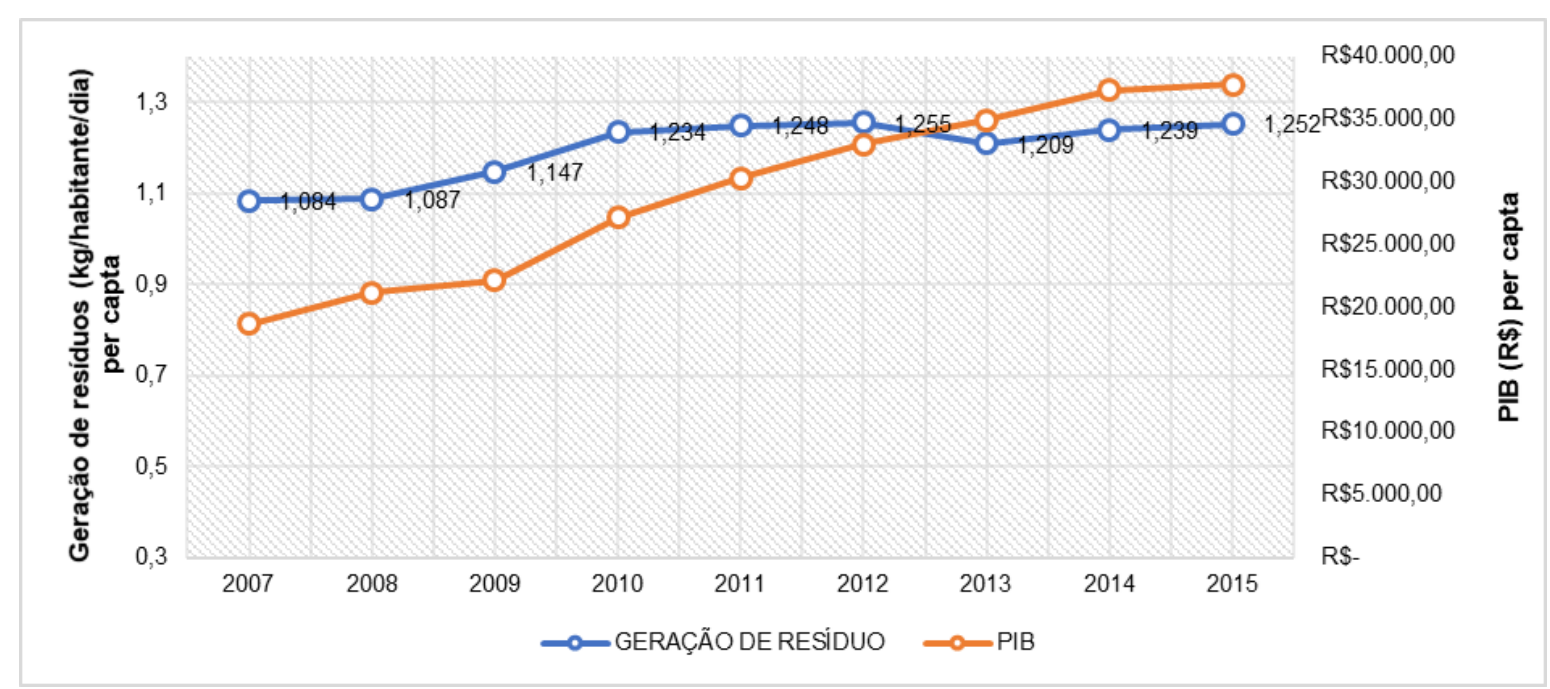

Fonte: Autores (2020).

Figura 12 - Análise de correlação entre a geração de resíduo x PIB entre os anos de 2007 e 2015 na região Sudeste: (a) Função de correlação entre as variáveis; (b) Grau de correlação.

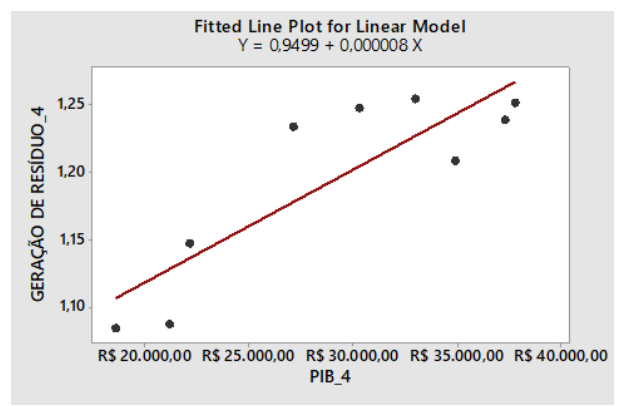

(a)

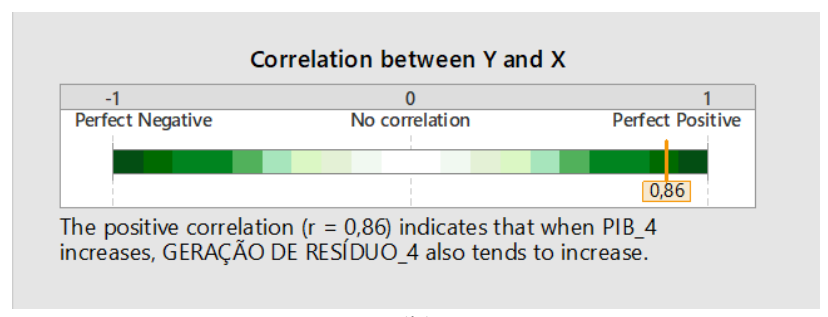

(b)

Fonte: Autores (2020).

A região Sudeste, indica uma correlação positiva dos parâmetros, tendo em vista o valor de correlação de 0,86 (Figura 12 , item $b)$.

\section{Região Sul:}

Quanto à região Sul do Brasil, segundo IBGE (2020), a região possui população de 30.192.315 habitantes e área de $576.774 \mathrm{~km}^{2}$. Segundo dados da PNUD (2018), a região possuía um IDH de 0,754, terceiro índice entre as cinco regiões do Brasil.

Os dados coletados quando ao PIB e geração per capita de resíduos são apresentados na Figura 13. Na Figura 14, seguem os resultados da análise de correlação. 
Figura 13 - Geração de resíduos per capita x PIB per capita na região Sul (2007 a 2015).

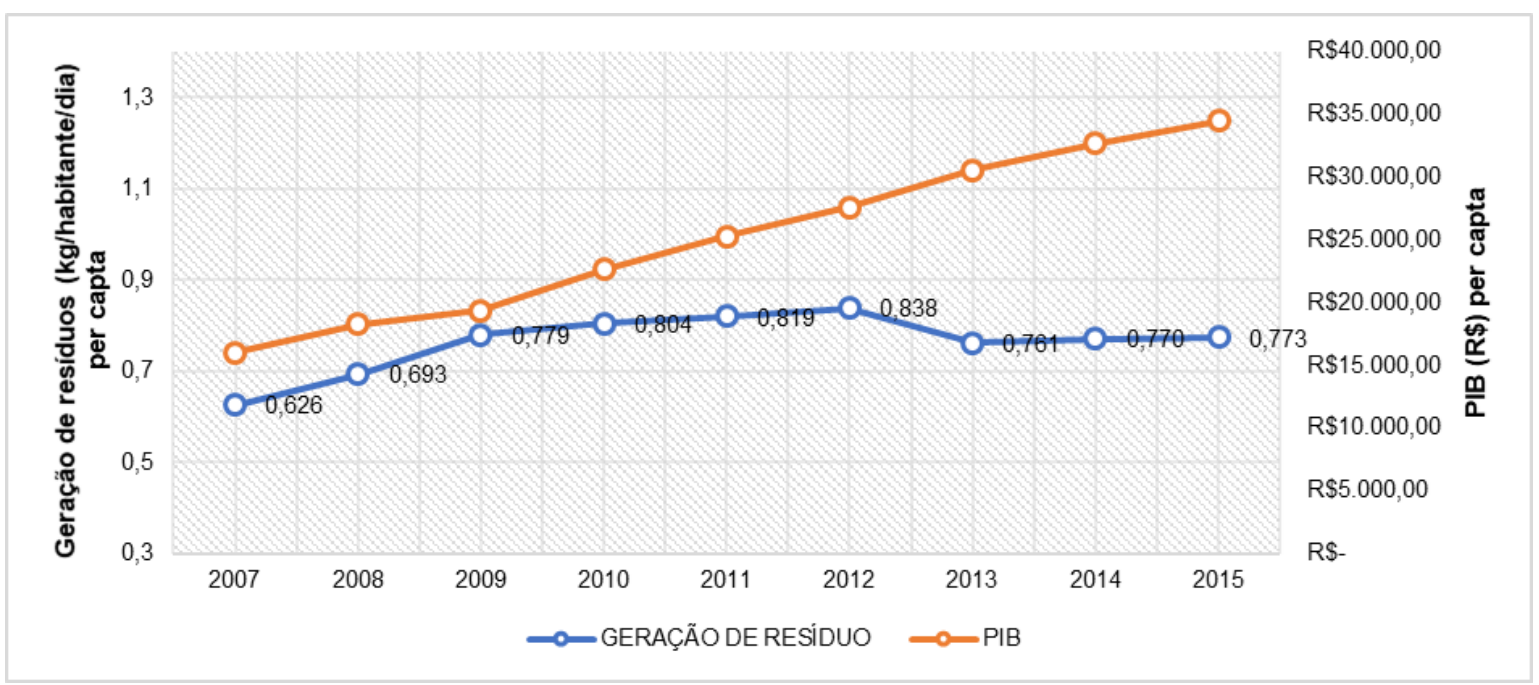

Fonte: Autores (2020).

Figura 14 - Análise de correlação entre a geração de resíduo x PIB entre os anos de 2007 e 2015 na região Sul: (a) Função de correlação entre as variáveis; (b) Grau de correlação.

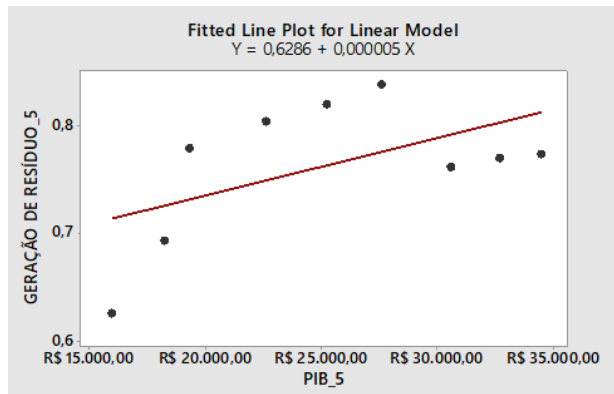

(a)

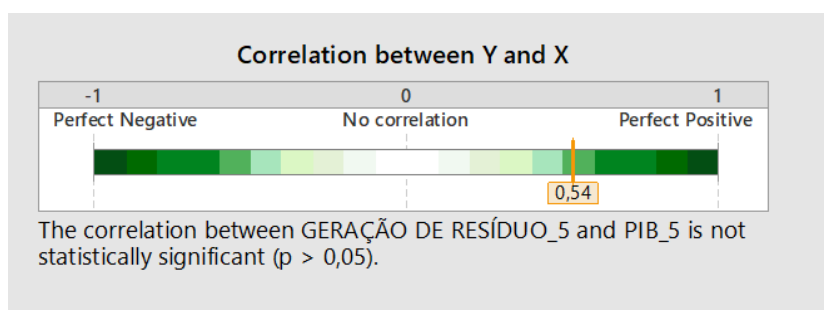

(b)

Fonte: Autores (2020).

Quanto à taxa de variação do PIB, percebe-se um crescimento acentuado ao longo do período de análise. A geração per capita foi crescente entre os anos de 2007 e 2012, caindo em anos seguintes (Figura 14, item a). Os dados mostram que, estatisticamente, não existe correlação entre os indicadores, com grau de correlação de 0,54 (Figura 14, item b).

\section{Análise comparativa:}

Na Tabela 1, é mostrado o resumo dos valores de correlações e os níveis de correlação de cada região estudada nesta pesquisa. 
Tabela 1: Resumo dos valores e níveis das correlações.

\begin{tabular}{ccc}
\hline Região & $\boldsymbol{\rho}(\mathbf{x , y})$ & Correlação \\
\hline Brasil & $-0,41$ & Baixa \\
Norte & $-0,38$ & Baixa \\
Nordeste & 0,75 & Alta \\
Centro-oeste & 0,80 & Alta \\
Sudeste & 0,86 & Alta \\
Sul & 0,54 & Média \\
\hline
\end{tabular}

Fonte: Autores (2020).

As análises entre as regiões, mostraram que três das cinco regiões do Brasil apresentaram taxa de correção entre PIB e geração de resíduos per capito, que foram: Nordeste, Centro-Oeste e Sudeste. As regiões Sul e Norte, assim como os dados nacionais não apresentaram valor de alta correlação entre as variáveis.

Segundo Marder et al. (2018) estudos obtidos com a observação do PIB per capita e sua relação com a geração de resíduos podem auxiliar administradores municipais a planejarem medidas que minimizem impactos ambientais e um melhor gerenciamentos dos resíduos.

\section{Conclusões}

O presente estudo teve como objetivo analisar a relação da geração de RSU per capita do Brasil com o seu Produto Interno Bruto (PIB), longo das duas primeiras décadas do século XXI. A partir dos resultados obtidos, que na maioria das regiões do país (Nordeste, Centro-Oeste e Sudeste) existe uma correlação alta entre a geração de resíduos sólidos e o PIB per capta, e que nas demais regiões, assim como na visão geral do país, essa correlação não se apresenta devido a influência de crises que se manifestaram ao longo dos anos.

Além disso, vale destacar a importância de tratar os aspectos de cada região, tendo em vista que tanto a extensão territorial, densidade populacional, aspectos culturais e principais práticas econômicas, as quais incidem no comportamento do PIB e da geração dos resíduos. Demanda-se uma análise cuidadosa dessas variáveis de forma a promover o desenvolvimento dessas regiões e conseguir implantar práticas sustentáveis.

Para trabalhos futuros, é sugerido a inserção de outras variáveis de análise que levem em consideração aspectos sociais e ambientais, de forma que sejam feitas novas correlações a fim de estimar as principais contribuições para a geração de RSU com o objetivo de minimizar a geração dos mesmos.

\section{Referências}

ABRELPE. (2017). Roteiro para encerramento de lixões. Associação Brasileira de Empresas de Limpeza Pública e Resíduos Especiais - ABRELPE. http://abrelpe.org.br/roteiro-para-encerramento-de-lixoes/.

ABNT. (2004). Resíduos Sólidos: classificação, NBR 10.004. Rio de Janeiro. Associação Brasileira de Normas Técnicas - ABNT.

Agência Brasil. (2019). Brasil gera 79 milhões de toneladas de resíduos sólidos por ano. Publicado no website da Agência Brasil. https://agenciabrasil.ebc.com.br/geral/noticia/2019-11/brasil-gera-79-milhoes-de-toneladas-de-residuos-solidos-por-ano.

Lei n. 12.305, de 2 de agosto de 2010. (2020). Diário Oficial da União, Brasília, DF. http://www.planalto.gov.br/ccivil_03/_ato20072010/2010/lei/l12305.htm.

Campos, H. K. T. (2012). Renda e evolução da geração per capita de resíduos sólidos no Brasil. Engenharia Sanitária e Ambiental, 17(2), 171-180.

Coutinho, T. (2017). Minitab: o que é e por que usar? Publicado no website do Minitab. https://www.voitto.com.br/blog/artigo/programa-minitab.

Sousa, C. A. F., Campos, J. C. B., \& De Oliveira, B. M. (2016). Panorama do gerenciamento dos Resíduos Sólidos no Brasil e no Nordeste após a implementação do PNRS. Revista Científica ANAP Brasil, 9(15). 
Deepask. (2015). Confira o Produto Interno Bruto - PIB por região do Brasil. Publicado no website Deepask - O mundo e as cidades através de gráficos e mapas. http://www.deepask.com/goes?page=Levantamento-mostra-como-o-PIB-esta-distribuido-entre-as-regioes-do-Brasil.

IBGE. (2010). Censo demográfico 2010. Instituto Brasileiro de Geografia e Estatística. https://censo2010.ibge.gov.br.

IBGE. (2020). Censo demográfico 2020. Instituto Brasileiro de Geografia e https://ftp.ibge.gov.br/Estimativas_de_Populacao/Estimativas_2020/serie_2001_2020_TCU.pdf.

Jucá, J. F. T. et al. (2014). Análise das diversas tecnologias de tratamento e disposição final de resíduos sólidos urbanos no Brasil, Europa, Estados Unidos e Japão. Recife: CCS Gráfica Editora Ltda.

Lakatos, E. M., \& Marconi, M. D. A. (2003). Fundamentos da metodologia científica. In Metodologia científica.

Marder, M. et al. (2018). Análise da influência do Produto Interno Bruto (PIB) e da população urbana na geração per capita de resíduos sólidos em municípios do interior do RS, Brasil. Revista Gestão \& Sustentabilidade Ambiental, 7(3), 21-35.

Martins, G. G., \& Stein, R. D. (2014) Panorama Setorial 2015-2018: Resíduos Sólidos Urbanos. Departamento de Meio Ambiente da Área de Meio Ambiente do BNDES. https://web.bndes.gov.br/bib/jspui/bitstream/1408/17680/1/PRCapLiv_residuos\%20solidos_compl_P.pdf.

Miragaya, J. F. G. (2014). O desempenho da economia na Região Centro-Oeste. Publicado no website do BNDS. https://web.bndes.gov.br/bib/jspui/bitstream/1408/7301/1/O\%20desempenho\%20da\%20economia\%20na\%20Regi\%C3\%A3o\%20Centro-Oeste_14_P.pdf.

Oliveira, B. O. S., \& Medeiros, G. A. (2019). Evolução e Desafios no Gerenciamento dos Resíduos Sólidos Urbanos nos Estados da Região Norte, Brasil. Revista Valore, 4(1), 749-761.

Pereira, A. S., Shitsuka, D. M., Parreira, F. B., \& Shitsuka, R. (2018). Metodologia da pesquisa científica [recurso eletrônico [eBook]. Santa Maria. Ed. UAB/NTE/UFSM. https://repositorio. ufsm. br/bitstream/handle/1/15824/Lic_Computacao_MetodologiaPesquisa-Cientifica. pdf.

PNUD. (2018). Ranking IDH Global 2018. Política das Nações Unidas Para o Desenvolvimento. http://www.br.undp.org/content/brazil/pt/home.html.

Resende, G. M., \& Magalhães, J. C. R. (2013). Disparidades do produto interno bruto (PIB) per capita no Brasil: uma análise de convergência em diferentes escalas regionais (1970-2008). Repositório do Conhecimento do Ipea, Brasília.

Silva, R. R., \& Caires, L. M. B. (2019). O potencial de mercado dos municípios da região Sudeste do Brasil: uma avaliação do índice de acessibilidade. Publicado nos anais do Encontro Nacional da Associação Brasileira de Estudos Regionais e Urbanos - ENABER. https://brsa.org.br/wpcontent/uploads/wpcf7-submissions/1107/Acessb_Sudeste_1.pdf.

Sjöström, M., \& Östblom, G. (2010). Decoupling waste generation from economic growth—A CGE analysis of the Swedish case. Ecological Economics, 69(7), 1545-1552.

Sousa, J. D., Lima, D. C., Da Silva B., F. C., De Medeiros, A. P., De Paiva, A. C. C., \& Maracaja, P. B. (2017). O desenvolvimento da região nordeste: uma abordagem econômica e ambiental. Revista Brasileira de Gestão Ambiental, 11(1), 42-48.

Spiegel, M. R., Schiller, J. J., \& Srinivasan, R. A. (2016). Probabilidade e Estatística-: Coleção Schaum. Bookman Editora.

Valor econômico. (2018). PIB per capita cresce 0,3\% em 2018, para R\$ 32.747. Publicado no website Valor econômico. https://valor.globo.com/brasil/noticia/2019/02/28/pib-per-capita-cresce-03-em-2018-para-r-32-747.ghtml. 